\title{
High Art Revisited: A Photogrammetric Approach
}

\author{
Lindsay MacDonald \\ Geomatic Engineering \\ University College London \\ ucfslwm@ucl.ac.uk
}

\author{
Ali H. Ahmadabadian \\ Geomatic Engineering \\ University College London \\ ali.ahmadabadian@ucl.ac.uk
}

\author{
Stuart Robson \\ Geomatic Engineering \\ University College London \\ s.robson@ucl.ac.uk
}

\author{
Ian Gibb \\ Conservation \& Collection Care \\ Historic Royal Palaces \\ ian.gibb@hrp.org.uk
}

\begin{abstract}
A photogrammetric technique was developed to monitor a painted heritage ceiling for evidence of cracking and flaking. A rig with up to four miniature cameras with lighting was raised $8 \mathrm{~m}$ on a telescopic mast to within $60 \mathrm{~cm}$ of the ceiling. Sets of images were analysed by a multiview stereo technique to extract point clouds from which a 3D representation of the surface could be obtained. The factor limiting the performance of the system was found to be not image processing but the mechanical characteristics of the telescopic mast.
\end{abstract}

Photogrammetry. Multiview stereo. Image processing. Conservation.

\section{INTRODUCTION}

The painted ceiling high above the Queen's Staircase at Hampton Court Palace is subject to cracking and the formation of paint flakes, and conservators are closely monitoring its condition. In a previous investigation a method was developed for 3D imaging of the ceiling, based on the principles of photometric stereo (MacDonald et al. 2012a; 2012b). A Nikon camera with a $300 \mathrm{~mm}$ telephoto lens was mounted on a tripod on the floor of the gallery, and a flash light was raised on an 8metre mast to cast a raking light across the surface of the ceiling (Fig. 1). By taking a series of images with illumination from different directions, we were able to demonstrate the feasibility of the technique to visualise the surface topography in small areas at a spatial resolution of $0.1 \mathrm{~mm}$.

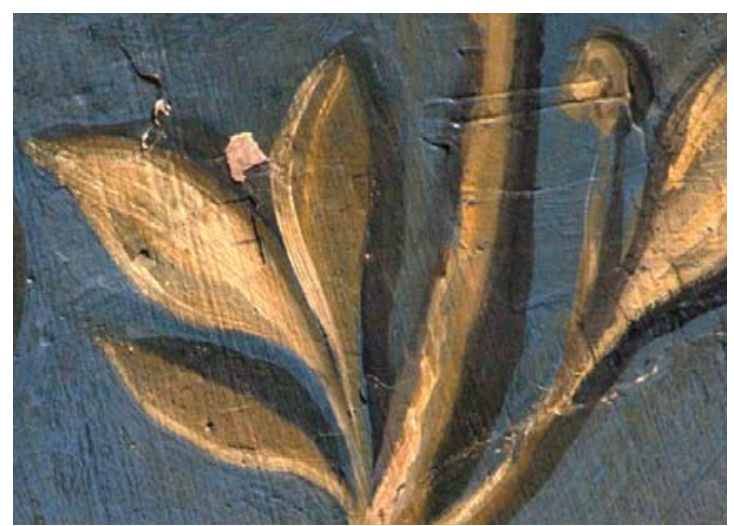

Figure 1: Detail of painted ceiling taken in October 2011 , illuminated by raking light, showing flakes and cracks. The camera was mounted on a tripod at floor level
In a further investigation during 2012 and 2013, a different approach was taken. Miniature monochrome cameras and LED lights were mounted on a horizontal bar, and raised by means of a telescopic mast to $60 \mathrm{~cm}$ below the ceiling. Images from the cameras were captured simultaneously by purpose-developed software in a laptop computer at floor level. A series of image sets was acquired at each location, rotating the mast between each set. From the image sets a dense and accurate point cloud was generated, using a photogrammetric bundle adjustment procedure, from which visualisations of the surface were obtained. The objective was to deploy the system at intervals of three months at the same locations to monitor the condition of the ceiling and detect any changes. In this paper we describe the four phases of the project, and the successive elaboration of the apparatus and refinement of the image processing methods in each phase.

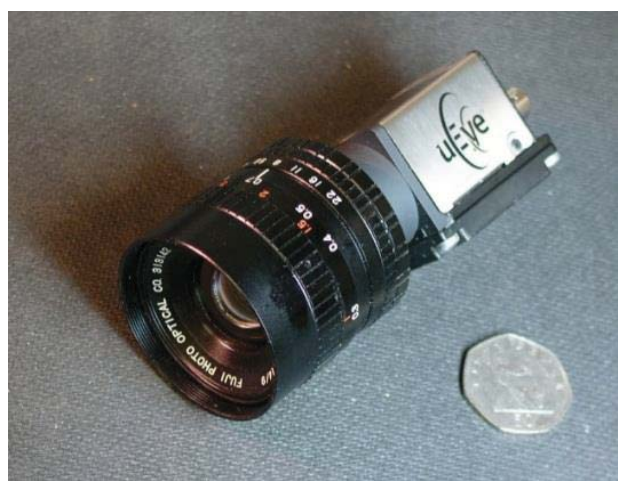

Figure 2: Miniature uEye camera fitted with Fujinon 9mm C-mount lens. The $50 p$ coin gives an indication of size 


\section{FIRST PHASE}

For the new project it was important to have lightweight cameras that could easily be operated at height. We chose the IDS UI 5480CP-M-GL camera (hereafter called uEye) because of its proven performance in other real-time photogrammetric and industrial applications (Fig. 2). It takes C-mount lenses and has a monochrome CMOS sensor of dimensions $5.6 \times 4.2 \mathrm{~mm}$ with pixel dimension of 2.2 microns, producing an image of $2560 \times 1920$ pixels in $4: 3$ aspect ratio with 12 bits per pixel. The camera has no Bayer mask or infrared cutoff filter, and so generates a monochrome image representing the integral of radiant power over the whole spectral range 400-1100 $\mathrm{nm}$. The camera weighs only $61 \mathrm{~g}$ and can be powered through an Ethernet cable.

Two uEye cameras were mounted on a horizontal aluminium bar with a distance of $60 \mathrm{~cm}$ between centres. Each camera was turned inwards by an angle of $20^{\circ}$. A miniature laser was fixed alongside each camera at the same angle so that its beam was parallel to the camera's optical axis. The two beams converged at a distance of $82 \mathrm{~cm}$ from the bar. The principle of operation was to raise the telescopic mast supporting the rig progressively towards the ceiling until the two laser spots converged, at which position the two cameras should produce sharp images (the lenses having been pre-focussed for this distance). Local illumination of the ceiling was provided by a battery-powered LED ring-light on an annular mount around the mast end-pole (Fig. 3).

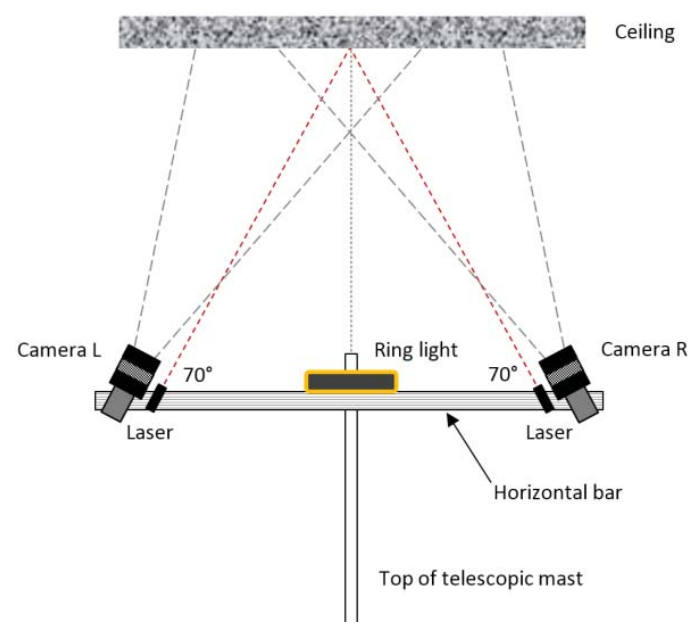

Figure 3: Arrangement of camera pair on top of telescopic mast, showing the lasers and overlapping fields of view

An 8-metre telescopic aluminium mast with its integral tripod was set up on the floor of the gallery, close to the top of the stairs (Fig. 4). Three 10metre cables were connected to the bar: two Ethernet for the cameras, plus a 6 volt supply for the lasers. The sections of the mast were successively raised by hand until the correct operating height was reached. The LED ring light cast sufficient illumination onto the ceiling for the sensitivity of the cameras. After each image pair was captured the mast was rotated by approximately $30^{\circ}$ in order to obtain a series of image pairs, with the objective of covering the same region of the ceiling from different angles.

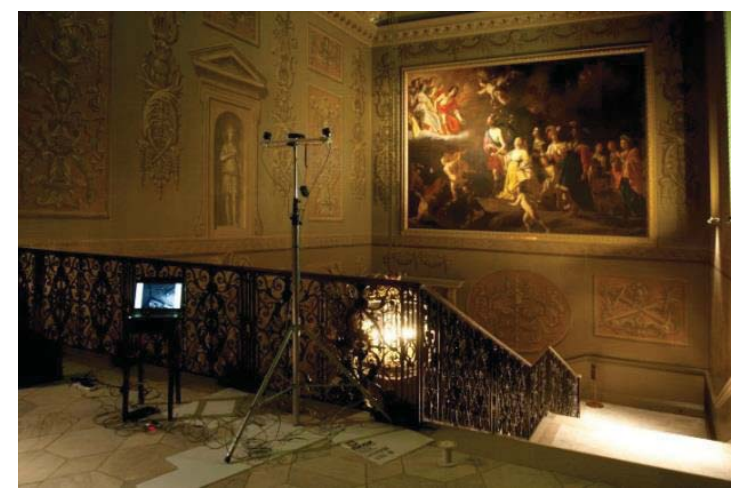

Figure 4: Location of aluminium mast on gallery floor

The lens used in this phase was the Fujinon Cmount $25 \mathrm{~mm} f / 0.85$ which gave superlative image quality. With the $1 / 2$ " sensor the lens had a field of view of $14^{\circ} 35^{\prime} \times 10^{\circ} 58^{\prime}$ and each image covered an area of $\sim 150 \times 115 \mathrm{~mm}$ on the ceiling. The area of overlap was approximately $1080 \times 1850$ pixels, corresponding to an area on the ceiling of $67 \times 115$ $\mathrm{mm}$ at a surface resolution of 16 pixels $/ \mathrm{mm}$. The lens aperture was $f / 8$ and exposure time 109 msec.
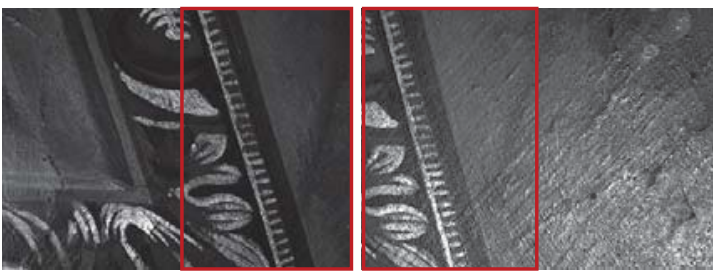

Figure 5: Images from left and right cameras showing the region of overlap

The laser positioning and image capture systems worked well, but the light-weight aluminium mast was not adequate. In order to obtain the required $3 \mathrm{D}$ resolution it is necessary to have at least 12 images of the same area. The main problem was that the mast at full extension was not exactly vertical but curved to one side by a few centimetres. As it was rotated for successive image pairs the laser spots did not remain stationary in the same position on the ceiling but followed a circular trajectory of radius approximately $20 \mathrm{~cm}$. The image pairs therefore did not afford sufficient overlap for the extraction of 3D surface data. In summary the aluminium telescopic mast was deemed inadequate to support the weight of the 
payload (approx. $1 \mathrm{Kg}$ ). Also the $25 \mathrm{~mm}$ lenses on the cameras produced too much magnification and insufficient overlap of the images.

\section{SECOND PHASE}

The general approach was the same as in the previous phase, but various changes were made to the equipment:

- A telescopic fibreglass mast was used, with five sections of $240 \mathrm{~cm}$ of diameter $57-30$ $\mathrm{mm}$. A semi-circular aluminium mounting bracket of $30 \mathrm{~mm}$ diameter was fabricated to clamp the camera bar.

- The mast was anchored at floor level by a heavy-duty steel tripod. It was steadied around the base section by a longer aluminium Leica survey tripod with a $58 \mathrm{~mm}$ collar at $1.8 \mathrm{~m}$ height.

- Two 500 watt tungsten-halogen spotlights were mounted on tripods at floor level to provide illumination on the ceiling in addition to the LED ring-light on the mast.

- Fujinon $9 \mathrm{~mm}$ lenses were fitted on the two uEye cameras to give a wider field of view and hence a greater area of image overlap.

- The dangling cables were secured with electrical tape to the mast to keep them close to the centre of gravity and minimise lateral forces on structure.

The new fibreglass mast used in the second phase was stronger and more stable than the previous aluminium mast, and the two tripods at the base provided good anchoring for the structure. However there was very little damping in the mechanical structure, and after any movement the camera rig tended to sway back and forth, continuing for up to a minute. While not dangerous, this resulted in significant motion blur of many of the images with the relatively long exposure time of $320 \mathrm{msec}$.

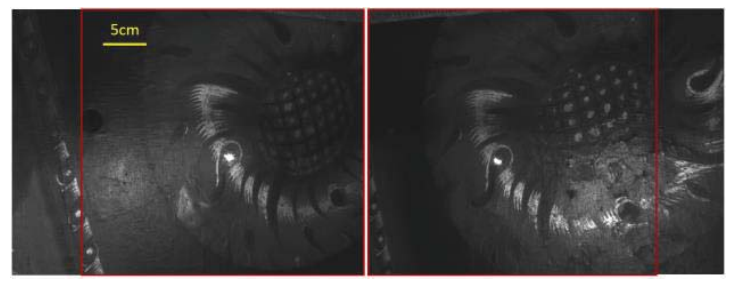

Figure 6: Images from left and right cameras with $9 \mathrm{~mm}$ lenses, showing the region of overlap of $31 \times 31 \mathrm{~cm}$. The laser beams appear as a bright spot near the centre

The two lenses were set to aperture $f / 11$ and focus distance $0.7 \mathrm{~m}$, and the outer rings taped to prevent movement. In the lab previously the rig had been set up to the laser convergence distance of $705 \mathrm{~mm}$ and the image area was determined to be $44 \times 33 \mathrm{~cm}$ with a region of overlap of $31 \times 31 \mathrm{~cm}$ (Fig. 6).
Because the cameras were tilted with respect to the surface, the depth of field was limited and the outer $5 \mathrm{~cm}$ on each side was out of focus. The resolution at the centre of the field was $\sim 5.8$ pixels $/ \mathrm{mm}$, i.e. each pixel covered 170 microns on surface. Illumination from the LED ring light positioned on the top of the mast was quite localised, resulting in considerable non-uniformity across the image area. A set of 22 image pairs was captured from a single mast location close to the handrail at the gallery centre. The mast was rotated by intervals of $\sim 18^{\circ}$ between successive positions.

The sets of image pairs were processed in an attempt to generate a dense 3D point cloud of the ceiling surface. The original images were analysed in VisualSFM to generate a dense point cloud. This software package includes bundler, cluster multiview stereo (CMVS) and patch-based multi-view stereo (PMVS). The resulting point cloud was too sparse to discriminate $3 \mathrm{D}$ features on the ceiling due to insufficient textured area in the images. In order to improve the quality of images, corrections were applied to the images to remove geometric distortion caused by the camera lens. These included optical aberration (radial and tangential lens distortions) and axial displacements of the lens wrt the sensor (offset of principal points from the image centre, affinity and orthogonality). Corrected images were again analysed in VisualSFM, and a photo-grammetric multi-view stereo method was applied to the corrected images to resolve the scale.

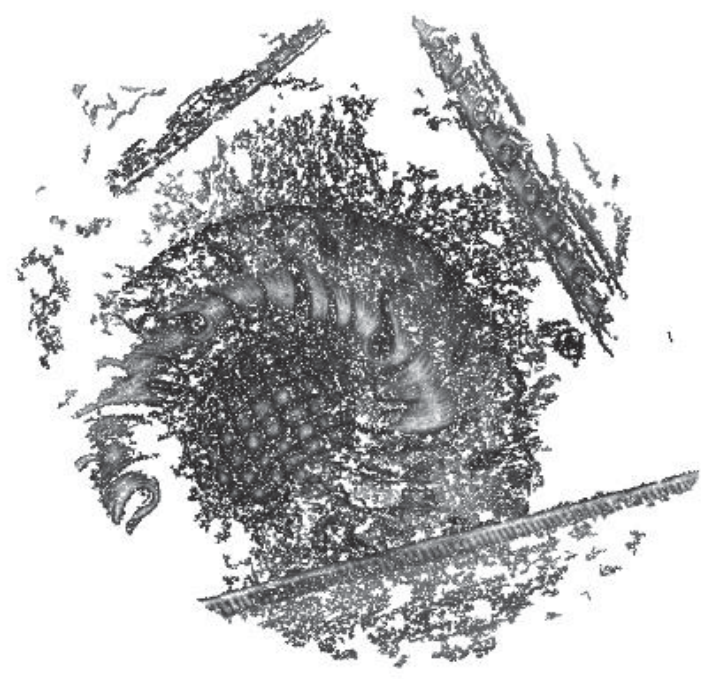

Figure 7: The images were corrected for geometry and radiometry and used in the process of $3 D$ point generation. Level 1 of image pyramid was used for $3 D$ reconstruction

Although this correction increased the number of points in the final point cloud, the results again showed that the quality of the images was insufficient to achieve a dense point cloud (Fig. 7). The result proved the importance of surface texture 
in generating a dense point cloud and in reducing the level of noise in the points. It was concluded that the more feature points are visible on the object, the more spatial detail and less noise there is in the final point cloud. Having evaluated the quality of images for $3 \mathrm{D}$ reconstruction, we tested the feasibility of using photogrammetric multi-view stereo software to obtain a dense and accurate point cloud. Bundler was run on the images to generate a sparse point cloud and estimate the exterior orientation parameters. These data were imported into the Vision Measurement System (VMS) software to run photogrammetric bundle adjustment to improve the precision. Unfortunately Bundler was not able to estimate the exterior orientation parameters accurately and the uncertainty in estimating the base line after resolving the scale was too high (around $5 \mathrm{~mm}$ ). This result showed the importance of image texture not only for dense point cloud generation, but also to resolve the scale accurately.

\section{THIRD PHASE}

Further modifications were made to the apparatus with the aim of reducing the blur and hence increasing the detail in the images:

- Two lightweight nylon cords were attached to the top of the mast and allowed to dangle down to floor level on each side, to dampen the swaying motion.

- Four 4.5W LED lamps (12 volt, MR16 sockets) were mounted on the camera bar (Fig. 8) to provide local illumination of the ceiling. Each lamp produced 360 lumens, cool white colour balance, with a beam angle of 60 degrees. These were powered from a 20W power supply at ground level via a twin cable up the mast.

- The weight of the augmented assembly, excluding trailing cables, was 1,600 grams.

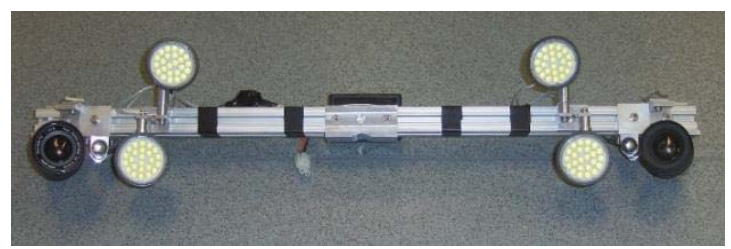

Figure 8: Top view of the camera rig in phase three, showing camera lenses, positioning lasers \& LED lights

Image sets were taken at two different locations on the ceiling above the gallery (Fig. 9). The first was a rosette design, the second a crossed ribbons motif. Tungsten halogen spotlights at floor level were employed to provide a broad circle of illumination in addition to the LED lights on the rig. The two 9mm Fujinon lenses were fixed at aperture of $f / 11$ and focus distance of $0.7 \mathrm{~m}$, same as for the second phase. Image pairs were taken at each location (Fig. 10) with floor spotlights alone and then supplemented by the LED lights. The exposure times ranged from 150 to $250 \mathrm{msec}$ for the spotlights alone, but were reduced to $52 \mathrm{msec}$ when the LEDs were switched on. This suggests that the illuminance of the ceiling from the LEDs (at a distance of approximately $60 \mathrm{~cm}$ ) was 3-4 times that of the spotlights (at a distance of $\sim 8$ metres).

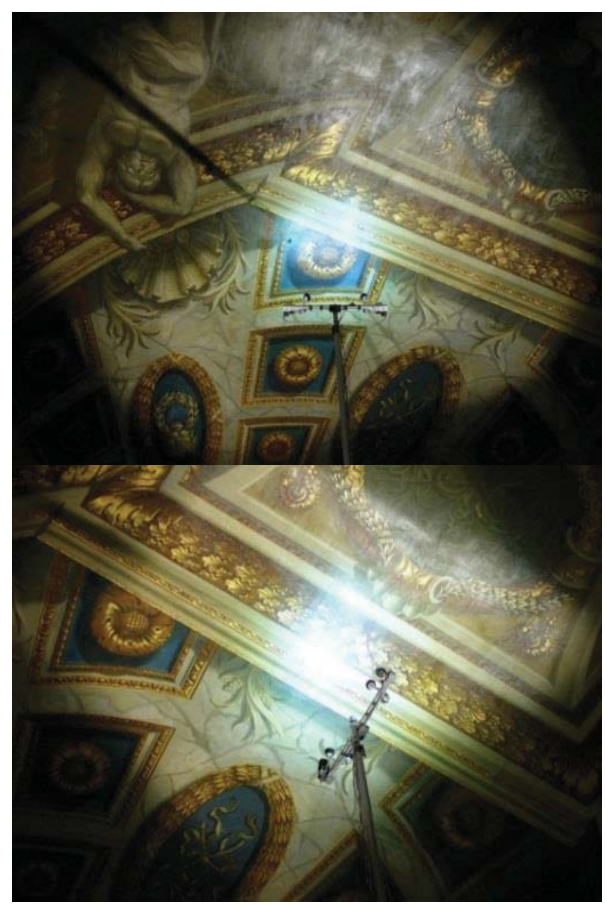

Figure 9: Mast deployed at two locations, showing the circle of yellowish light on the ceiling from floor-mounted spotlights and the localised bluish illumination from the four LED lights on the mast head

In each lighting condition at each location 16 to 18 image pairs were taken, rotating the mast by approximately 20 degrees between each. The time interval between successive images was approximately 2 minutes, so a full set of images from one mast location took about 30 minutes.
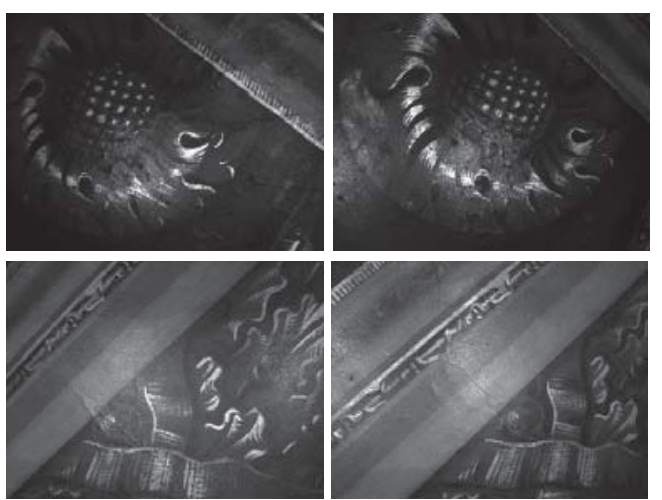

Figure 10: Pairs of images from left and right cameras under LED lighting at the first location (top) showing a flake and the second location (bottom), showing a crack 
From each set of images a dense and accurate point cloud was generated, by a method (Fig. 11) similar to that presented by Ahmadabadian et al. (2013). After geometrically correcting ('undistorting') the images, corresponding measurements extracted from the images were used to compute approximate $3 \mathrm{D}$ coordinates in Bundler.

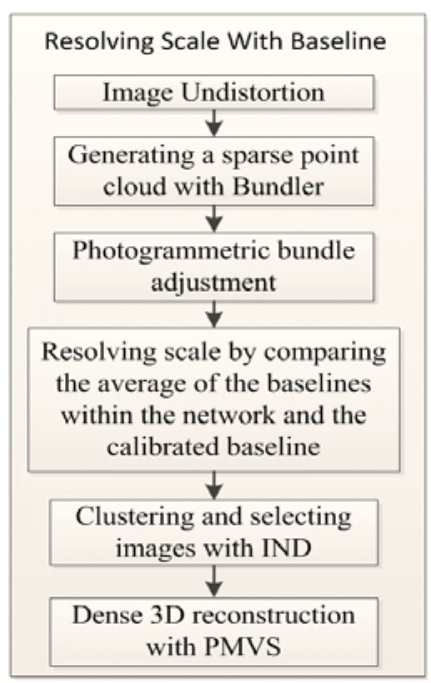

Figure 11: Generating a point cloud from the image set

A photogrammetric bundle adjustment with relative orientation parameters of the stereo camera pair as geometric constraints was run on the data to estimate the length of the stereo baseline within the network. This length was compared with the calibrated baseline to estimate a scale factor, which was applied to camera locations and 3D coordinates. After resolving the scale, these data were input into the PMVS processing software to generate a dense point cloud.

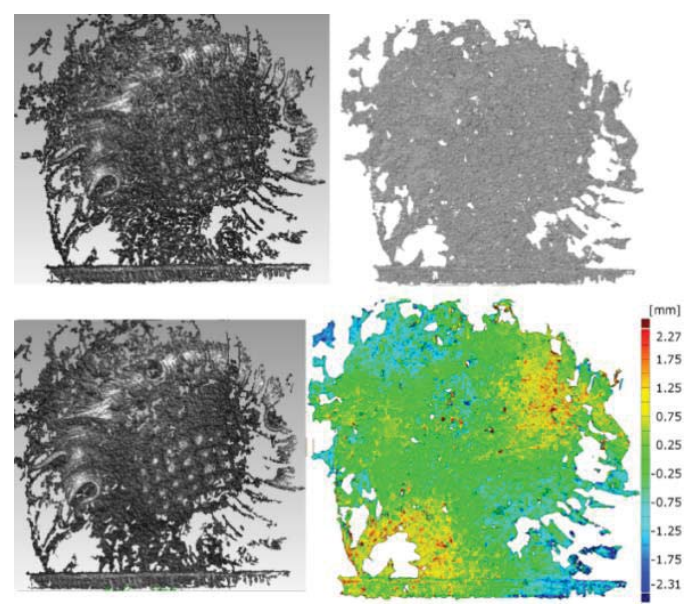

Figure 12: The point cloud (top-left), mesh (top-right), textured mesh (bottom-left) and deviation from a plane fitted to point cloud (bottom-right) for the first location

The results of the 3D processing (Fig. 12) showed that an elevation map could be obtained from the point cloud representation, revealing salient surface features. The pock marks in two regions of the first location and the crack in the second location were clearly visible (Fig. 13), with a depth resolution of $\sim 0.25 \mathrm{~mm}$ (i.e. 250 microns).

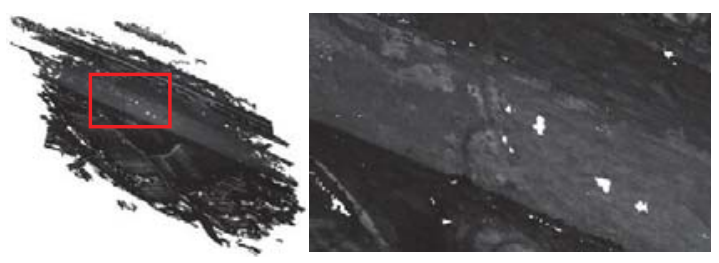

Figure 13: (left) Point cloud for the second location on the ceiling, tilted; (right) enlarged detail showing crack

At this resolution the spacing of the points was barely sufficient to resolve the structure, and the point cloud still had many holes. In order to achieve the objective of resolving features of 100 microns, higher image magnification would be necessary. This could be achieved only by using lenses of longer focal length, brighter lights and shorter exposure times. It was noted during image analysis that images taken under tungsten halogen light alone were less sharp than those where the LED lights were also on. This was attributed to the relatively poor optical performance of the lenses at infrared wavelengths, with chromatic aberration causing point spread and image blur.

\section{FOURTH PHASE}

Further changes were made to the camera rig (Fig. 14 ) with the aim of enhancing image quality:

- Two additional uEye cameras were added to the rig. The outer two retained Fujinon $9 \mathrm{~mm}$ lenses (same as in phases two and three) but the inner two had Zoomar Macromar 36mm f/2.8 lenses.

- Infrared cutoff filters were fitted to all lenses, $43 \mathrm{~mm}$ diameter on the outer and $52 \mathrm{~mm}$ on inner.

- Four additional LED lamps were added, of the same type as before. The total light output of the eight LEDs was increased to 2880 lumens, requiring a new $36 \mathrm{~W}$ power supply.

- All components were positioned so that the centre of gravity was as close as possible to the central axis of the supporting mast (in order to minimise bending moments).

- The previous two conventional heavy Ethernet cables were replaced by four lightweight Cat 6 ribbon cables (10m length), one for each camera.

The advantage of using uEye cameras powered via Ethernet is that up to eight can be added to the same controller (Netgear gigabit hub) as 'plug and play'. 


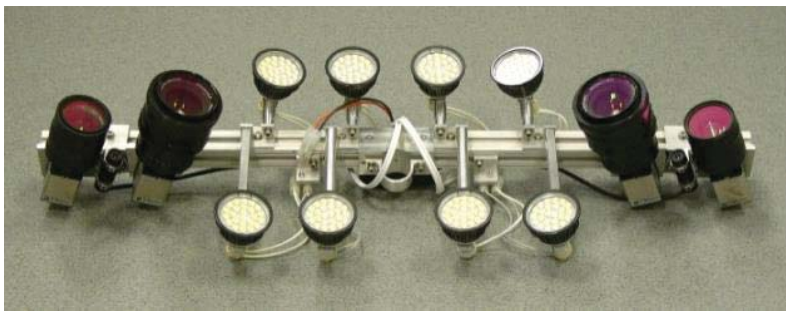

Figure 14: View of the modified camera rig in phase four, showing four cameras with lenses in two symmetrical pairs, two positioning lasers and eight LED lights

Other modifications were made to the mast, with the aim of improving stability:

- The legs of the aluminium Leica survey tripod were fitted with metal studs, inserted into three holes in the horizontal floor supports of the heavy-duty steel tripod. Together these formed a rigid structure for anchoring the mast base (Fig. 15).

- Thicker nylon ropes were draped on either side to enhance dampening of the mast swaying motion.

- The weight of the complete rig as payload (excluding trailing cables) was 2,760 grams.

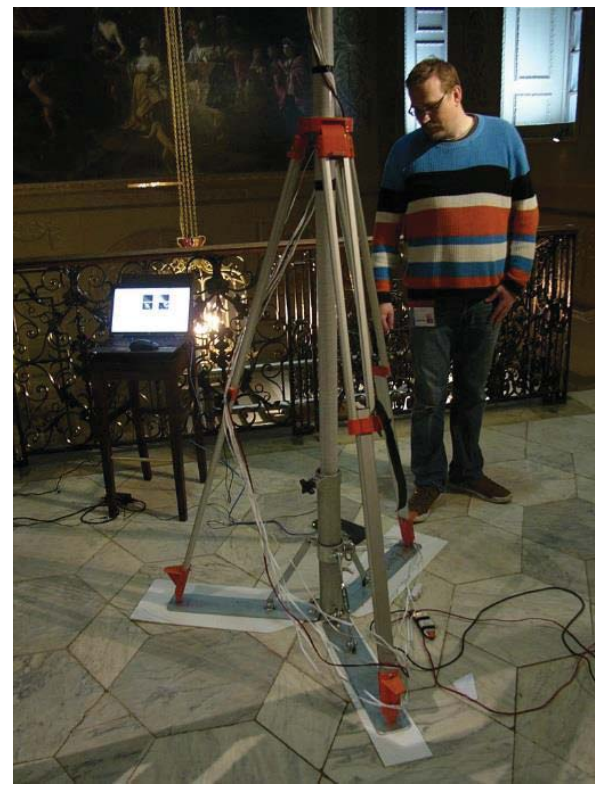

Figure 15: Tripod support of mast base, deployed on the marble floor of the gallery

The general approach was the same as in the previous phases, using convergence of the beams of the two lasers to set the correct operating height of the mast so that the camera bar was $\sim 60 \mathrm{~cm}$ from the ceiling (Fig. 16). The floor-to-ceiling height was measured to be $8.52 \mathrm{~m}$, so the exact height of the bar was $7.92 \mathrm{~m}$ above the floor. Image sets were taken at three different locations on the ceiling above the landing, two the same as for the previous visit (rosette and crossed ribbons) plus a third in the vicinity of a newly missing paint flake. All four lenses were fixed at aperture $f / 11$ and focus distance of $0.65 \mathrm{~m}$. The VMS uEyeCapture software on the laptop computer displayed live views of images from all four cameras. At each click four monochrome images were captured simultaneously and stored in 8-bit TIFF format. In prior testing in the lab at UCL the image scale was established: for the telephoto $36 \mathrm{~mm}$ lenses the surface area was $83 \times 62 \mathrm{~mm}$, corresponding to 31 pixels $/ \mathrm{mm}$ (pixel size $=32$ micron), and for the $9 \mathrm{~mm}$ wide-angle lenses the surface area was $410 \times 295 \mathrm{~mm}$, corresponding to 6.5 pixels $/ \mathrm{mm}$ (pixel size $=150$ micron).

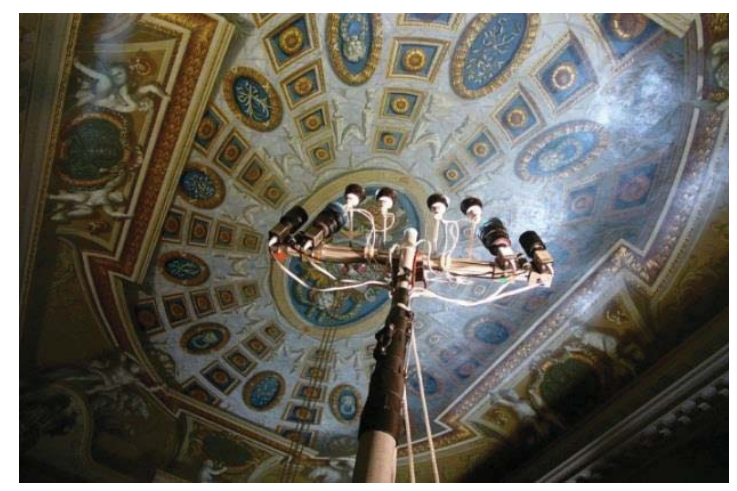

Figure 16: Rig in phase four, attached to mast with all cables connected, ready to be raised

Exposure times for all images from the ceiling were fixed at $47 \mathrm{msec}$ for the outer cameras (wide-angle $9 \mathrm{~mm}$ lenses) and $23 \mathrm{msec}$ for the inner cameras (36mm lenses). At each ceiling location eight image sets were taken, rotating the mast by approximately $45^{\circ}$ between each position.
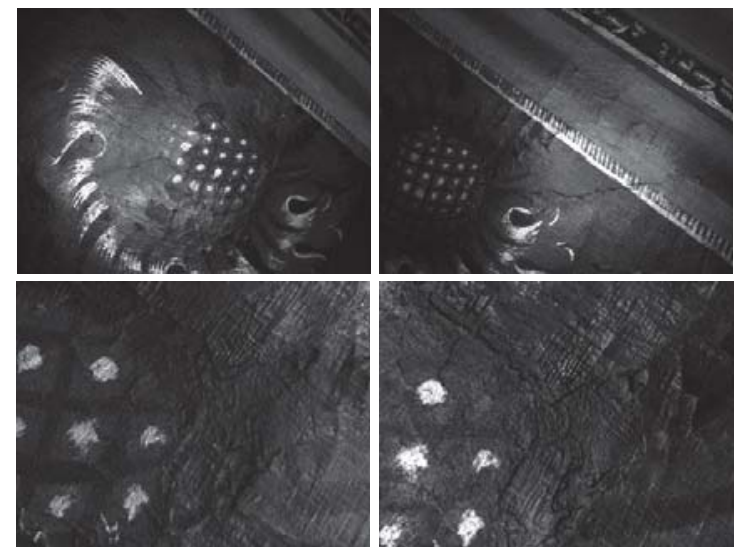

Figure 17: A set of four images at the first ceiling location, showing (top) left and right views through the wide-angle $9 \mathrm{~mm}$ lenses and (bottom) left and right views through the telephoto $36 \mathrm{~mm}$ lenses

Each image set consisted of three successive exposures producing four images each, so a total of $12 \times 8=96$ images was captured for each ceiling location. The average time between exposures following each mast rotation was $\sim 4$ minutes, 
allowing a period for the oscillation of the mast to settle. The images were sharp and in clear focus (Figs. 17-19), showing an abundance of both painted detail and surface texture. Old losses of paint and repainted areas were clearly visible, and also cracks in the surface.
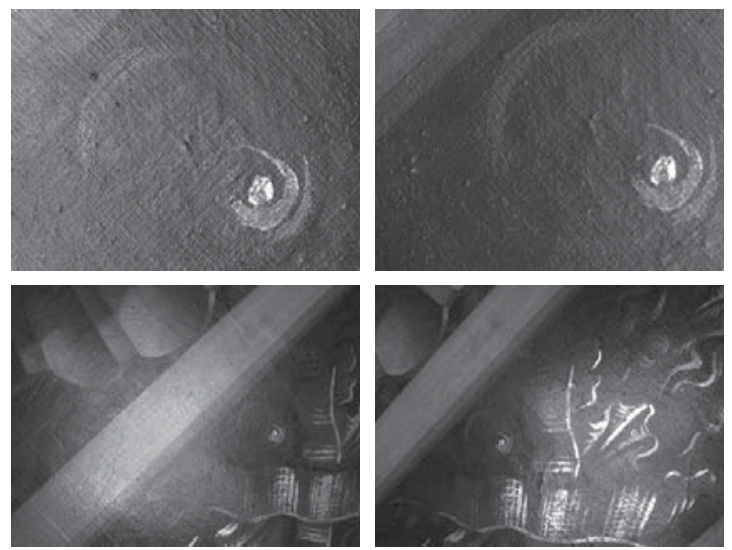

Figure 18: A set of four images at the second ceiling location, showing (top) left and right views through the wide-angle $9 \mathrm{~mm}$ lenses and (bottom) left and right views through the telephoto $36 \mathrm{~mm}$ lenses

The two telephoto images covered almost exactly the same area of the ceiling (about the size of a credit card), whereas the two wide-angle images (each about the size of an A3 sheet of paper) covered rather different areas horizontally because of the geometry of their overlap. The changes made to the apparatus resulted in much better image quality: the images from this phase were mostly sharp and free from blur.
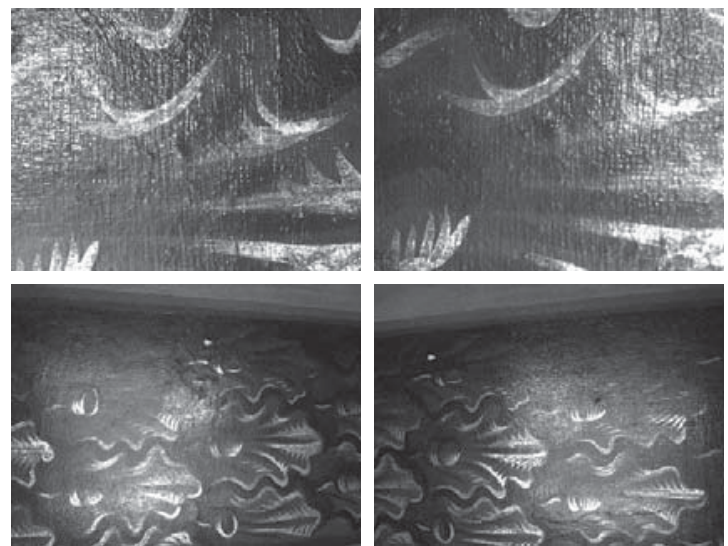

Figure 19: A set of four images at the third ceiling location, showing (top) left and right views through the wide-angle $9 \mathrm{~mm}$ lenses and (bottom) left and right views through the telephoto $36 \mathrm{~mm}$ lenses

Processing the image sets followed the same procedure as before, except that now there were two concurrent sets of images. The aim was to use the outer low-resolution set from the cameras with $9 \mathrm{~mm}$ lenses to establish the scale, and the inner high-resolution set from the cameras with $36 \mathrm{~mm}$ lenses to extract fine surface details.

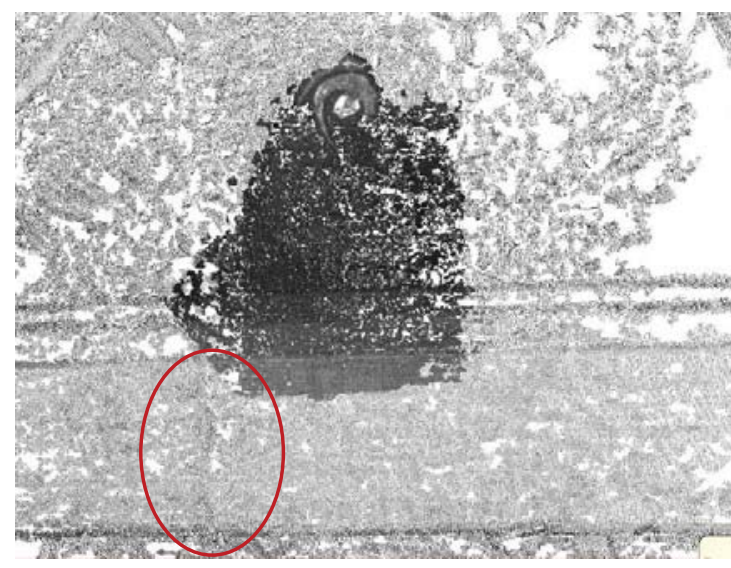

Figure 20: Point cloud extracted from images at the first ceiling location. The region of interest is in the red ellipse

When the images were processed, however, it was found that in rotating the mast there was still some eccentricity, which caused the image region captured by the inner cameras at high magnification to move in a circular locus on the ceiling. Because the same area was not covered in all image pairs, the resolution of the point cloud was insufficient to show the surface features to the required accuracy (Fig. 20).

\section{CONCLUSIONS}

The original objective of the project was to employ digital imaging methods to monitor the fragile condition of the painted ceiling at intervals of three months. But at the end of this long and complicated project, which continued for over 18 months, we had to confront the unhappy truth that we had failed to develop an apparatus and method robust enough to provide repeatable image sets of specified locations on the ceiling. We believe that the photogrammetric processing workflow can generate point clouds of sufficiently high resolution if it is given image sets of high quality and sufficient overlap. The problem for us has been in expecting too much of minimal structures to support the camera rig.

The new fibreglass mast turned out to be more difficult to deploy than the aluminium mast for four reasons, which collectively may have implications for health and safety:

- It was heavier to carry to the deployment site, especially the steel base tripod;

- The mast section length of 2.4 metres meant that it was necessary to stand on an adjacent step ladder in order to raise, turn and lower the telescopic sections. This was stressful when working near the gallery rail and drop beyond; 
- It took considerable strength to lift the final combined weight of just under $3 \mathrm{Kg}$ for the camera rig, plus $1 \mathrm{Kg}$ for the cables, plus 5 $\mathrm{Kg}$ for the inner sections of the mast (i.e. nearly $9 \mathrm{Kg}$ in total), especially when standing on a step ladder and lifting from chest height.

- Turning the mast for successive image pairs required climbing the step ladder, loosening the clamp at the top of the lowest (outermost) section of the mast, turning the inner section and the whole structure above while not changing the height, then relocking the clamp. Although not simple, this proved to be less difficult than raising the mast.

The biggest challenge faced with this technique was the physical handling and erection of the mast. At the operating height of 7.9 metres (Fig. 21), and with the head weight of approximately $2.8 \mathrm{Kg}$ (the same as our laptop computer), the mast was only just stable with the limited footprint of the tripod arrangement at the base (Fig. 15). Even small variations of a few millimetres in the height of the marble floor tiles caused the mast to lean away from vertical. Turning the mast for successive image sets then caused the top of the mast to move in a circular locus. Also it was very difficult to predict the exact region of the ceiling that would appear in the image frame when the mast was fully raised, so it was not really practicable to target specific features. This made it nearly impossible to achieve the intended purpose of monitoring the same region in repeated visits at three-monthly intervals.

To implement the method successfully would require a more substantial mast with the following characteristics:

- Broader base and better fixings of the tripod legs (or other bracing means) to achieve more stability of the whole structure;

- Mechanism to raise and lower the sections of the mast, either by manual crank or electrical motor;

- Fine X-Y positioning mechanism for the head carrying the camera rig, so that it could be manoeuvred into the desired position relative to the ceiling without moving the base;

- Mechanism to rotate the mast about its axis, preferably by electrical means, by a given angle;

- Stiffer structure with dampening to minimise the swaying and oscillation of the cameras.

The ideal would have been the robotic system developed in the VITRA project (MacDonald 2006).

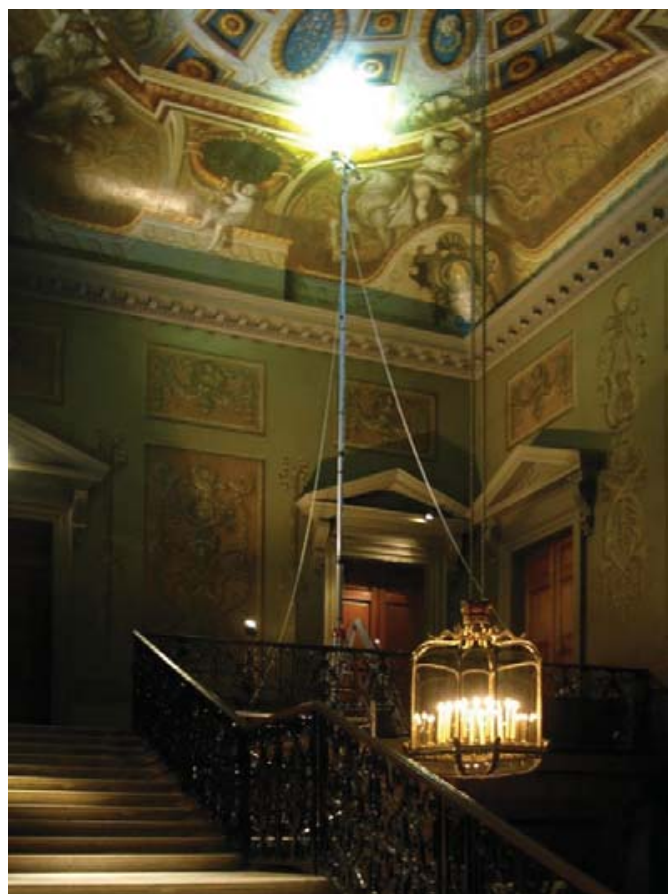

Figure 21: Mast fully raised to $8 m$ above the gallery with the photographic rig operating $60 \mathrm{~cm}$ below the ceiling

\section{ACKNOWLEDGEMENTS}

This project was supported in part by a small research grant from Historic Royal Palaces. Thanks to John Hindmarch for able assistance on site during the final visit to Hampton Court Palace. Thanks also to lan Seaton (UCL Engineering) for fabricating various mechanical components, without which our rig would have not attained the heights.

\section{REFERENCES}

Ahmadabadian, A. H., Robson, S., Boehm, J., Shortis, M., Wenzel, K., and Fritsch, D. (2013). A comparison of dense matching algorithms for scaled surface reconstruction using stereo camera rigs. ISPRS J. Phot. \& Remote Sensing, 78, 157167.

MacDonald, L. W. (2006) A robotic system for digital photography. Proc. SPIE Conf. on Digital Photography II, Vol. 6069.

MacDonald, L. W., Gibb, I., and Robson, S. (2012a) Conservation Monitoring of a Heritage Ceiling by Photometric Stereo. Proc. XXII ISPRS Congress, Melbourne.

MacDonald, L. W. Gibb, I., Robson, S., and Vlachou-Mogire, C. (2012b) High Art: Visualising Damage on a Heritage Ceiling. Proc. Conf. on Electronic Visualisation and the Arts (EVA), London, 309-316. 